\title{
The Effect of Proof Format on Reading Comprehension of Geometry Proof: The Case of Indonesian Prospective Mathematics Teachers
}

\author{
Lathiful Anwar ${ }^{1,2^{\star}}$, Angeliki Mali ${ }^{1}$, Martin J Goedhart ${ }^{1}$ \\ ${ }^{1}$ Institute for Science Education and Communication, University of Groningen, Nijenborgh 7, 9747 AG Groningen, \\ NETHERLANDS \\ 2 Department of Mathematics, Faculty of Mathematics and Natural Sciences, Universitas Negeri Malang, Jl. Semarang No.5, \\ Malang, East Java 65145, INDONESIA
}

Received 25 June 2020 • Accepted 8 February 2021

\begin{abstract}
This study aims to investigate the effects of the use of multiple geometry proof formats on Indonesian students' reading comprehension of geometry proof (RCGP). Four classes of prospective secondary mathematics teachers $(\mathrm{N}=125)$, aged 18 to 19 years, participated in this quasi-experimental study. While the experimental group was instructed in three proof formats (paragraph, two-column and flow-chart proof), the control group was instructed in only the twocolumn proof format. Similar pre- and post-tests, based on Yang and Lin's (2008) RCGP test, were administered to both groups. N-Gain scores were used to determine the improvement of both groups. The N-Gain scores showed significantly more improvement of students' RCGP in the experimental group. More detailed analysis indicated that the use of multiple proof formats supports the students' understanding of the facets of logical status of statements and the critical ideas in the proof. This study shows the benefits of offering multiple proof formats to support prospective mathematics teachers' RCGP.
\end{abstract}

Keywords: geometry proof, reading comprehension, proof formats, flow-chart proof, pre-service teachers

\section{INTRODUCTION}

Most of the geometry teaching in Indonesian secondary schools focuses on introducing properties of geometric figures such as similarity and congruence of polygons (e.g., triangle, rectangle, etc.) and their application in measurement problems (Ministry of Education and Culture, 2013, 2016; National Education Standard Board, 2020). In Indonesia, geometry proof is formally introduced in the first year at the university level, specifically in the study programs of mathematics and mathematics education. One of the objectives in learning geometry proof is that students are able to understand and construct proofs and apply them in other contexts.

The first author is a lecturer in Euclidean Geometry for Indonesian prospective mathematics teachers (PMT) (aged 18-19 years) at a teacher education university in Indonesia. At this university, students observe in class how teachers present a proof, read proofs in their mathematics textbooks and construct their own proof of theorems and geometric propositions. Through these activities, students are expected to comprehend proofs. Like in most countries, proof comprehension along with proof construction, proof validation and proof evaluation are expected learning outcomes at the university level (Selden, 2012).

However, based on the first author's small-scale observational study in geometry classrooms, most of the PMT faced difficulties with the understanding of the written proofs in the textbook or the lecture notes. These difficulties were more frequent when the proofs were written in the two-column format, which is the usual format in the country. For instance, students had problems with identifying propositions which support or justify intermediate or final conclusions. Through this study we aim at finding ways to improve teaching geometry proof to these prospective mathematics 


\section{Contribution to the literature}

- This study investigates and then justifies experimentally the recommendation of previous theoretical studies that the use of multiple proof formats supports students' reading comprehension of geometry proof.

- This study suggests the use of the flow-chart proof format together with other formal proof formats (i.e., paragraph and two-column proof) for the comprehension of geometry proof; the flow-chart can visualize the logical connection of the components of the proof which cannot be provided by other formats.

- $\quad$ This study also suggests combining reading-oriented tasks and writing-oriented tasks to support students' reading comprehension.

teachers in the Indonesian context by comparing the effects of research-informed teaching design and regular teaching.

A number of studies investigated the role of proof format in the presentation of proofs in geometry, such as flow-chart and two-column proof (e.g., Cirillo \& Herbst, 2011; Miyazaki et al., 2014, 2017). The literature study by Cirillo and Herbst (2011) suggested that the use of multiple formats, namely paragraph, two-column, tree and flow-chart proof, could support students' comprehension of geometry proof. Research findings from Miyazaki et al. $(2014,2015,2017)$ showed that using the flow-chart proof format to construct a geometry proof could help grade 8 students (aged 13-14 years) understand the elements of proof and their logical relationship.

Therefore, we conjectured that the use of multiple proof formats might improve the reading comprehension of geometry proof of the PMT. In this paper, we report on a quasi-experimental study in order to investigate the role of multiple formats in supporting PMT reading comprehension of geometry proof, aiming at helping Indonesian PMT overcome their difficulties with comprehension of geometry proof. We start this paper with presenting the existing literature in reading comprehension, then we present information about the context of the study, our methods, the findings, and we conclude with recommendations for teaching geometry proof and future research.

\section{THEORETICAL BACKGROUND}

\section{Aspects of Proof}

Based on the research literature, Selden and Selden (2017) distinguished four interrelated aspects of proving, namely proof comprehension, proof construction, proof validation and proof evaluation. Proof comprehension concerns reading comprehension of proof written in textbooks or lecture notes, or demonstrated during lecture (Mejia-Ramos et al., 2012). Proof construction is the reasoning from proven facts (premises) using appropriate properties (e.g., definition, axioms, proven theorems) and logically valid steps to arrive at a conclusion (Knuth, 2002). Proof validation refers to "the reading of, and reflection on, a proof attempt to determine its correctness" (Selden \& Selden, 2017, p. 341 ), and proof evaluation is "making value judgments about a finished proof text" (Ibid, p. 341). Proof validation and proof evaluation relate to determining the correctness of proofs. However, proof evaluation concentrates on features of proofs including clarity, context, convincingness, beauty, elegance, and depth (Inglis \& Aberdein, 2015). For instance, the beauty of proof relates to simplicity, that is the number of accepted statements logically connecting premises and conclusion. And the elegance of proof relates to how the proof is presented (Rota, 1997). Selden and Selden (2017) suggested that students should have a good grasp of proof comprehension, proof construction and proof validation before attempting to evaluate proofs as beautiful, elegant, etc. Additionally, Selden and Selden (2017) recommended that the first two concepts: proof comprehension and proof construction should be taught together. So, as a starting point to learn or introduce proof, we consider that students' proof comprehension could benefit from attempts in proof construction.

\section{Models of Reading Comprehension of Geometry Proof}

In the context of teaching proof, proof comprehension refers to students' understanding of proof written in the textbook, in lecture notes, or demonstrated during a lecture. Development and refinement of research models of proof comprehension are essential for the development of research surveys and interview schemes, and for the design and evaluation of teaching interventions. In this section, we discuss some studies that developed such models of reading comprehension in the context of plane geometry with the aim to present the model used in our study.

Yang and Lin (2008) conceptualized a model of reading comprehension of geometry proof (RCGP), which is widely used in literature. They distinguished four levels of RCGP, as shown in Figure 1. The first level (Surface) relates to the understanding of particular statements, terms, symbols or figures in the proof. The second level (Recognizing Elements) refers to the logical status of statements in the proof. The third level 


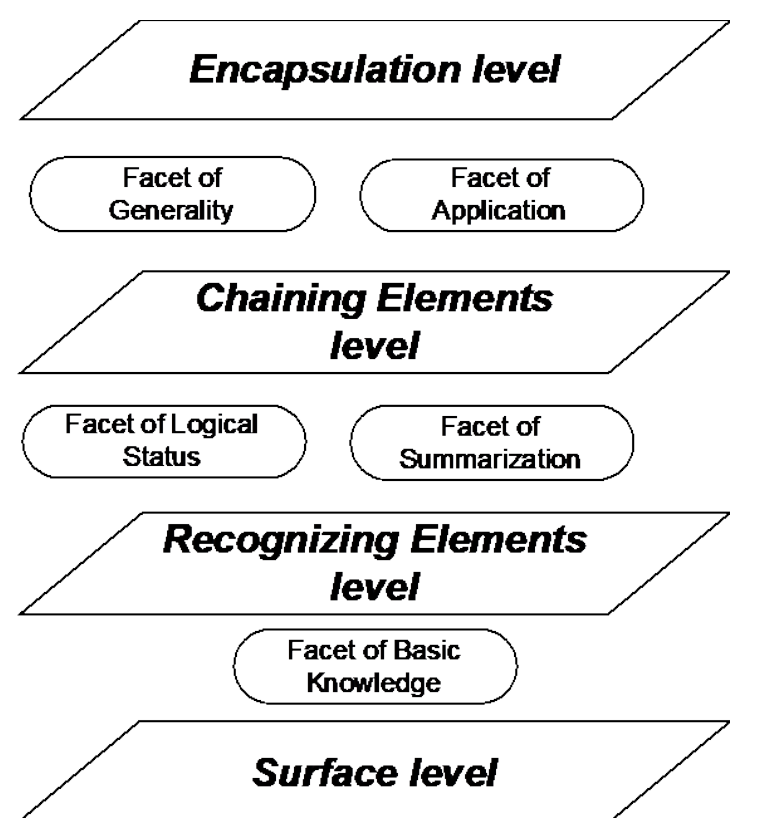

Figure 1. The five facets of RCGP (Yang \& Lin, 2008)

(Chaining Elements) is about comprehending and combining logical arguments or connections between statements. The last level (Encapsulation) concerns reflection on the proof as a whole in terms of main ideas and methods and the application of the proof in other contexts.

Yang and Lin (2008) also distinguished facets to describe steps of switching between the four levels of reading comprehension. The facets occur as passages between two adjacent levels as shown in Figure 1; thus, one who understands the related facet can move to the next level. For example, the facet of basic knowledge refers to an understanding of mathematical terms, figures and symbols stated in a proposition and its proof. This facet (basic knowledge) is needed to move from Surface level to Recognizing Elements level. The facet of logical status relates to the ability to correctly recognize the status of statements including premises, conclusions or applied properties in a proof. The facet of summarization is about identifying the core of proof or the critical ideas of proof. The two facets (logical status and summarization) are needed to step from Recognizing Elements level to Chaining Elements level. The facet of generality regards recognizing accuracy of a proposition and identifying what is validated by the proof. The facet of application is concerned with applying the proposition in another context. These two facets (generality and application) are needed to switch from the Chaining Elements level to the Encapsulation level.

Yang and Lin (2008) formulated operational definitions of the five facets of reading comprehension from existing literature and from interviews with five mathematicians and four mathematics teachers. They structured these facets using a hypothetical model. Then, they justified their hypothetical structure of the facets of reading comprehension by investigating students' performance on the facets of RCGP. The students were junior high school students in grade $9(\mathrm{n}=223)$ and senior high school students in grade $10(\mathrm{n}=378)$. Yang and Lin (2008) validated the hierarchical nature of their model through multi-dimensional scaling (MDS) of the data collected from these students' performances. They used the MDS analysis to construct a spatial structure representing the cognitive relations between the facets of the RCGP. They used two dimensions in their analysis because they assumed that the facets of RCGP contained two main components: relevant knowledge and logical reasoning. Based on their MDS analysis, they found that the structural relationship of the five facets of RCGP was sustained. Yang and Lin (2008) argued that in theory students' trajectory of reading comprehension would follow their hierarchical model.

However, Lin and Yang (2007) found an alternative way of students' development of RCGP. They found students starting from Surface and then to Encapsulation while skipping the Recognizing Elements and Chaining Elements levels. This indicates that students understand the terms and symbols in the proof, which proposition is validated by the proof and how to apply the proposition in other contexts, but they lack the recognition of logical status of the statements in the proof and the critical ideas of the proof. They also found that geometric knowledge of description and translation play an important role in RCGP. Description refers to verbal descriptions of geometrical concepts and properties, for instance how to read geometric symbols, like $\overline{A B} \cong \overline{B C}$, and how to explain this symbolic expression. Translation is associated with translating verbal descriptions into aspects of figures, for instance drawing a geometric figure that represents a proposition. This finding suggests that students need to have enough geometric knowledge, especially in terms of description and translation, before they can learn geometry proofs, and more specifically before they read a geometry proof.

Mejia-Ramos et al. (2012) created a model of proof comprehension, which refined and extended Yang and Lin's (2008) model focusing on undergraduate mathematical proof. They distinguished two levels of comprehension, namely a local and a holistic comprehension level. The local comprehension addresses the understanding of one or a small number of statements within the proof, while the holistic comprehension addresses understanding of the proof as a whole.

Even though the model by Mejia-Ramos et al. was developed for undergraduate proof, which fits to our participants, we used and adapted Yang and Lin's model in this current study to assess the prospective mathematics teachers' performance of RCGP. Firstly, we consider that the components of Yang and Lin's model are relevant to the learning goals of the geometry proof course for the Indonesian PMT, which are concerned 
with the meaning of the statements within the proof such as premises, intermediate conclusion and conclusion, and logical structure of proof. Secondly, the background of our participants' experience in proving was similar with Yang and Lin's participants who started to learn mathematical proof for the first time.

\section{Teaching Reading Comprehension of Geometry Proof}

In this section we review literature on students reading strategies and reading comprehension of proof, both at the university level and, if relevant, the secondary level. Studies on the identification of reading strategies are from different areas, like calculus and number theory (Weber, 2015) and geometry (e.g., Yang, 2012). Other studies investigate students' reading behaviour in comprehending and validating proofs (Panse et al., 2018). The previous literature also investigates methods to teach mathematical proof at university level (e.g., Roy et al., 2010). In this section, we detail some of these studies in order to situate the RGCP model and our study in the existing research literature.

Roy, Alcock, and Inglis (2010) compared students' reading comprehension of proofs in three conditions: (1) written textbook proof, (2) live lecture: proof presented by a lecturer in a standard undergraduate class, (3) a computer-based e-proof. They examined the undergraduate students' reading comprehension using Yang and Lin's (2008) proof comprehension model. The findings showed that the live lecture was most effective in improving undergraduate students' proof comprehension and the e-proof was least effective.

Yang (2012) investigated the use of cognitive and metacognitive strategies by grade 9 students (aged 14 to 15 years) to improve their proof reading. These two strategies referred to an explanation by Pereira-Laird and Deane (1997) that

metacognitive strategies involve planning, monitoring, and regulation activities that take place before, during and after any thinking activities such as reading. In contrast, cognitive strategies, refer to integrating new material with prior knowledge. Cognitive strategies that students use to acquire, learn, remember, retrieve and understand the material while reading include rehearsal, elaboration, and organizational strategies (p. 190).

Their findings showed that the use of metacognitive reading strategies directly influenced students' RCGP. Specifically, this study suggested that metacognitive reading strategies can be used for planning and monitoring comprehension of logical coherence and cognitive reading strategies for elaborating proof. In the current study, we introduced both strategies: cognitive and metacognitive strategies when students read a proof during the teaching intervention. The cognitive strategies focused on elaborating (i.e., reading proposition, hypothesizing and drawing inferences from a figure, reading to see the proof steps), and the metacognitive strategies focused on planning (i.e., skimming through or reading step-by-step) and monitoring (i.e., reflecting on how proof begins and comes to the conclusion, considering the relation between steps).

A study conducted by Yang and Lin (2012) compared the effects of reading-oriented tasks (proof comprehension) and writing-oriented tasks (proof construction) on 14-to-15-year-old students' RCGP. The experimental group was instructed to read and discuss reading tasks (reading-oriented tasks). In contrast, the control group was instructed to prove and apply the same propositions (writing-oriented tasks). They compared the scores of the post-test and the delayed post-test with the pre-test scores as covariates. Their research findings showed that students from the experimental group had better reading comprehension at a delayed post-test than those from the control group. And the experimental group's scores on all facets of reading comprehension except for the facet of application were significantly higher than those of the control group for both post-test and delayed post-test. However, the RCGP score of both groups at a delayed post-test were slightly lower than at the post-test. Thus, the reading-oriented tasks and writing-oriented tasks did not improve students' RCGP in the long run. Yang and Lin suggested that alternative approaches are needed to support students' reading comprehension, for instance, combination of both reading-oriented and writing-oriented tasks.

Weber (2015) observed four successful mathematics students in reading proofs of six theorems in introductory calculus and basic number theory. The study showed that there are five effective strategies used by successful mathematics students: (1) trying to prove a theorem before reading its proof, (2) identifying the proof framework being used in the proof, (3) breaking the proof into parts or sub-proofs, (4) illustrating difficult assertions in the proof with examples, and (5) comparing the methods used in the proof with student's own approaches. This study also surveyed 83 mathematics lecturers questioning them whether they desired their students to use these strategies. The data analysis showed that lecturers desired their students to use these strategies in an attempt to help them comprehend proofs. The author thought that the first strategy, namely trying to prove before reading its proof, is suitable for students who are able to construct a proof, but for the beginning prover this strategy is difficult.

Panse, Alcock, and Inglis (2018) investigated whether reading a mathematical proof on analysis and number theory for validation or comprehension engenders different processes. They analyzed the behavior of 16 mathematicians and 16 undergraduate students, using 
eye movement. They found nonsignificant differences between reading for comprehension versus reading for validation. These findings suggested that lecturers could choose between validation and reading comprehension tasks because these would not lead to a different reading behavior. In addition, a study on proof validation by Weber (2009) revealed that students sometimes successfully judged the validation of proofs even when they realized that they did not understand the proofs. Therefore, in the beginning of our teaching intervention, we focus on reading comprehension tasks.

Based on findings of empirical studies described in this section, we identified some factors that could foster students' reading comprehension of proof such as students' reading strategies and teaching method (e.g., live lecture). Effective reading strategies include metacognitive (e.g., planning, monitoring, and regulation), cognitive strategies (e.g., rehearsal, elaboration, and organizational strategies) and other five reading strategies (i.e., trying to prove before reading, identifying proof framework, breaking the proof in parts, using examples, comparing proof methods). We integrated these factors in the teaching design we used in our study to explore the role of different proof formats. These proof formats will be discussed in the following section.

\section{Different Formats of Presenting a Geometry Proof}

Mathematical proof, including geometry proof, is a special text genre in written discourse and students' ability to read a mathematical proof is required for understanding proof written by lecturers or from textbooks. A mathematical proof may be presented as a direct proof; that is a proof shown to be true directly as a result of other statements and situations that are true. Direct proofs apply deductive reasoning, which is the reasoning from proven facts (premises) using logically valid steps to arrive at a conclusion. There are four formats for presenting a geometry proof: paragraph proof, two-column proof, tree proof and flow-chart proof, as shown in Figure 2.

The paragraph proof consists of a detailed narrative, explaining the proof process including steps and reasons that lead to the final conclusion. The paragraph format helps students describe the logical chain of reasoning in a narrative style. A paragraph proof could support students to communicate effectively on a technical level, both verbally and in writing (Brandell, 1994).

The two-column proof consists of two columns where the first column contains a chronological list of statements leading to the desired conclusion. The second column contains a list of reasons supporting each step in the proof. These reasons could be, for example, axioms, definitions, and theorems. The two-column proof format helps students learn how to prove and what to use in their proof (Herbst, 2002).
A tree proof is a hierarchy of nodes connecting premises to a desired conclusion (Wong, Yin, Yang, \& Cheng, 2011). The tree proof consists of leaf nodes and derived nodes. Each node represents a step in the proof and each leaf node is a given statement or intermediate statement/conclusion. The tree proof explicitly visualizes graphically the logical relation between premises, intermediate propositions and conclusions, thereby facilitating students to determine what the given premises are or what conclusion is proven (Cirillo \& Herbst, 2011). However, the tree proof does not visualize the reasons supporting or justifying each statement.

The flow-chart proof displays the delineated structure of a proof using boxes (rectangles and rounded rectangles) and connecting arrows. The rectangular boxes contain premises, intermediate statements/conclusions and the conclusion, and the rounded rectangles contain axioms, theorems and definitions. A flow-chart proof represents deductive connections of premises and desired conclusions by identifying singular propositions (premises/prior statements and intermediate conclusion/conclusion) and universal propositions or supporting reasons (axioms, theorems and definitions). The flow-chart proof shows a storyline of proof: it starts with premises from which a conclusion is deduced, includes the theorems, axioms or definitions being used, and shows how the premises and a conclusion are connected (Miyazaki et al., 2015). This proof format is a good starting point for students to learn other formats of proof such as the paragraph and two-column proofs.

A theoretical study by Cirillo and Herbst (2011) offered proof tasks and discussed how multiple geometry proof representations move students toward more authentic proof practices. They proposed that the four proof formats (two-column, paragraph, tree and flow-chart proof) could support students' understanding and writing of geometry proof. They suggested that teachers introduce and give opportunities to students to use different types of reasoning and formats of proofs because using a single type of reasoning or format may obstruct students' creativity of reasoning and their understanding of the proof. However, a practice-oriented study is needed to verify this conjecture. Also, each proof format has advantages and disadvantages. In the context of reading comprehension, students might identify the elements of proof, connections between propositions, and reasons justifying the propositions when they read a proof in the two-column format. However, this may lead students to believe that the deductive process is more linear than it actually is. In contrast, the flow-chart proof uses the same statements and reasons as a two-column proof, but the logical flow connecting the statements is indicated by arrows (Miyazaki et al., 2015). A paragraph proof describes the logical argument using sentences and it looks more as "an explanation than a structured 


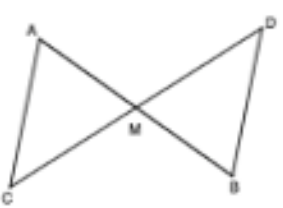

In $\triangle A M C$ and $\triangle B M D$, because $\overline{A M} \cong \overline{B M}$ and $\overline{C M} \cong \overline{D M}$ and because $\angle A M C \cong$ $\angle B M D$, then $\triangle A M C \cong \triangle B M D$, axiom S-AS. So, $\angle M A C \cong \angle M B D$, congruent polygons (corresponding angle).

(a) Paragraph proof

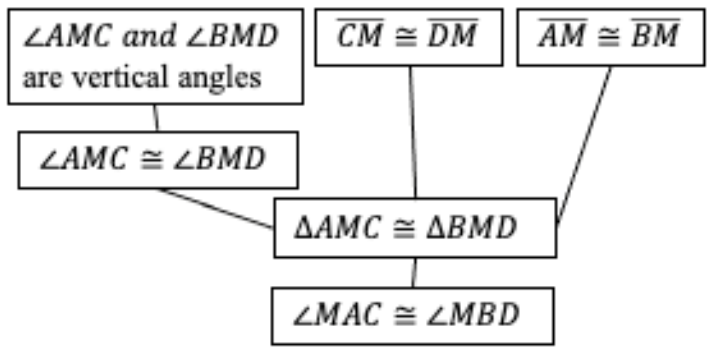

(c) Tree proof

Figure 2. Four different formats of geometry proof

mathematical devise" (Ibid., p. 25). This format could help students develop mathematical literacy and write a proof by contradiction. However, the lack of structure could be a problem in reading and writing a paragraph proof. The tree proof is a hierarchy of nodes leading from premises to conclusion (Wong et al., 2011), explicitly visualizing the logical relation between the elements of a proof. So, the tree proof could support students in determining what the given premises are or what conclusion is proved. However, the tree proof does not visualize the reasons justifying the validity of each statement.

The current study, in response to the theoretical study by Cirillo and Herbst (2011), will investigate the conjecture that multiple proof formats could foster students' proof comprehension. In this study, we introduced three formats of geometry proofs: paragraph, two-column and flow-chart proofs in the experimental group. This choice was made for several reasons. First, the paragraph and two-column proof formats are used in the textbook and lecture notes. Second, the flow-chart proof format has a feature that is not provided by the other proof formats, because it visualizes the connection between premises, intermediate conclusions, conclusion and reason/applied properties such as theorems, definitions, and axioms (Miyazaki et al., 2015, 2017). Third, we do not use the tree proof because this format is similar with the flow-chart proof (Cirillo \& Herbst, 2011).

\section{Aims of This Study}

The purpose of the current study was to investigate the effects of a teaching design that presents geometry proofs using multiple proof formats (two-column, paragraph and flow-chart proof) to support prospective mathematics teachers' RCGP. We implemented the design in an experimental group and compared their RCGP with a control group. In the experimental group, the students were asked to write a two-column proof or paragraph proof based on the flow-chart proof, and vice versa. In contrast, in the control group, proofs were presented in one format, the two-column proof when the students read and constructed geometry proofs during the lessons.

The corresponding research questions for this current study are:

1. Do students in the experimental group perform better at the post-test on RCGP than students in the control group?

2. Do students in the experimental group reach higher RCGP levels at the post-test than students in the control group?

3. Do students in the experimental group perform better at the post-test on each facet of RCGP than students in the control group?

\section{METHOD}

This current study investigates the impact of using multiple proof formats to foster RCGP of prospective mathematics teachers (PMT) by comparing their RCGP 
performance in a quasi-experimental pre-test/post-test design. The experimental group and the control group had six meetings of a geometry proof course with the control group using the two-column proof and the experimental group using three different proof forms (paragraph, two column and flow-chart proof). In the next sections we provide information about the context and the participants of the study, the instruction, the preand post- tests and the method used for data analysis.

\section{Context and Participants}

The participants of this study were PMT during the first semester of a four-year degree program. These PMT were students in a bachelor's degree program in a public teacher education institute (TEI) in Indonesia. The program required four years of coursework in both subject matter and pedagogical content, and an internship. After completion of this program, graduates could be mathematics school teachers. They were selected by schools as school-hired teachers or by district authorities as contract teachers or after a national test as civil servant teachers.

Four classes of in total 125 PMT (18-19 years old) who took an "Introduction to Geometry" course participated in this study. In this course, the PMT started learning formal geometry proofs. At their secondary school, they were introduced to the basic concepts of plane geometry such as properties of polygons (triangles, rectangle, square, etc.), similarity and congruence applied in measuring and calculation problems, but they were not introduced to proving. Two classes were randomly assigned to either the experimental group or the control group. In order to construct and read geometry proofs, two classes $(n=60)$ in the experimental group were instructed to work with three different proof formats, while the other two classes $(n=65)$ were instructed with the regular proof instruction of the two-column proof format.

Three lecturers participated in this study. The first author (ten years of teaching experience) taught the two classes in the experimental group, while two other lecturers (two years and twenty years of teaching experience) each taught one class in the control group. The first author is male and had seven years of experience in teaching geometry at university level. The two lecturers were female and had, respectively, one year and fifteen years of teaching experience in geometry.

\section{Instruction}

In the experimental group, the PMT took part in a sixmeeting teaching experiment that addressed geometry proof constructions and proof comprehension, with each meeting lasting for 150 minutes. In the first three meetings, the teaching focused on an understanding of definitions of geometric concepts (e.g., midpoint,
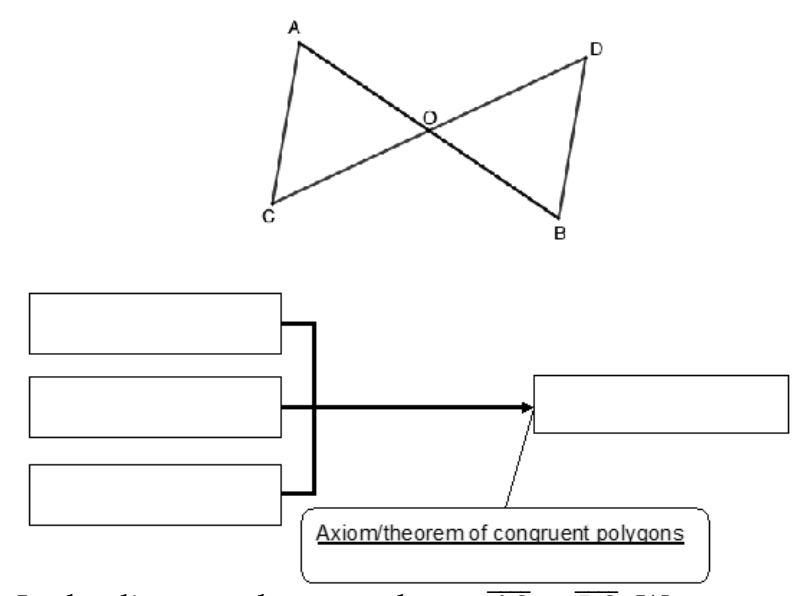

In the diagram above, we know $\overline{A O} \cong \overline{B O}$. We want to make $\triangle A C O$ and $\triangle B D O$ congruent. Which angles and sides should be congruent and what condition

(Axiom/Theorem) of congruent triangles should be used? Complete the flow-chart!

Figure 3. Open proof problem

bisector of a segment, bisector of an angle, vertical angles, etc.), axioms and postulates, and conjectures of geometric statements based on a step-by-step construction using dragging modalities of the GeoGebra Geometry application.

The last three meetings focused on reading and constructing geometry proofs. In the fourth meeting, the PMT constructed flow-chart proofs in open problems, such as determining possible conclusions when premises are given and determining other appropriate premises when a conclusion and one of the premises are given. The term 'open' refers to a situation where PMT could construct more than one suitable proofs. For instance, in the open problem as shown in Figure 3, PMT were given a conclusion of proof in a flow-chart proof format, and they were asked to determine the suitable statements to fill in the blank boxes of the flow-chart so that the proof is complete. In the fifth meeting, PMT read given flow-chart proofs, then converted these into a paragraph proof and a two-column proof. Finally, in the sixth meeting, PMT constructed a flow-chart proof of a geometric proposition, then converted the flow-chart proof into a paragraph and a two-column proof.

In the control group, during the six meetings, the lectures focused on introducing definitions of geometric concepts (e.g., midpoint, bisector of a segment, bisector of an angle, vertical angles, etc.), axioms/postulates and theorems through a teacher-centered pedagogy (talk and chalk) that used power-point presentations and the blackboard to construct geometric figures and to talk about the meaning of geometric concepts. The lecturers also introduced and demonstrated two-column proofs of theorems or propositions and asked PMT to read twocolumn proofs, and construct proofs in the two-column format. So, all proofs introduced by the lecturers and proof problems solved by PMT were written in the twocolumn format. 
Table 1. Structure of Reading Comprehension of Geometry Proof (RCGP) test (adapted from Yang \& Lin, 2008)

\begin{tabular}{|c|c|c|c|c|c|}
\hline Facets & Object of comprehension & Operational definition & Items & Score & Max score \\
\hline \multirow[t]{3}{*}{$\begin{array}{l}\text { Basic } \\
\text { knowledge (B) }\end{array}$} & Content of premises or conclusion & $\begin{array}{l}\text { Recognizing the meaning of a symbol in } \\
\text { the figure }\end{array}$ & 1 & 0,1 & 4 \\
\hline & & Explaining the meaning of a property & 2 & $0,1,2$ & \\
\hline & & Recognizing the meaning of a property & 3 & 0,1 & \\
\hline \multirow{3}{*}{$\begin{array}{l}\text { Logical status } \\
\text { (L) }\end{array}$} & Status of premise & Recognizing a condition applied directly & 4 & 0,1 & 3 \\
\hline & $\begin{array}{l}\text { Logical relation between premise and } \\
\text { conclusion }\end{array}$ & Judging the logical order of statement & 5 & 0,1 & \\
\hline & $\begin{array}{l}\text { Property applied to derive a conclusion } \\
\text { from premise }\end{array}$ & Recognizing which properties are applied & 6 & 0,1 & \\
\hline & Multiple arguments and critical ideas & Identifying critical ideas of a proof & $7-a, 7-b$ & 0,$1 ; 0,1$ & 2 \\
\hline \multirow[t]{2}{*}{ Generality (G) } & Proposition or proof & Judging the correctness & 8 & 0,1 & 3 \\
\hline & All arguments and attached figure & Identifying what is validated by the proof & $9-a, 9-b$ & 0,$1 ; 0,1$ & \\
\hline \multirow{2}{*}{$\begin{array}{l}\text { Application } \\
(A)\end{array}$} & Application in other situations & Applying the same premises & 10,11 & 0,$1 ; 0,1$ & 4 \\
\hline & & Identifying the different premises & 12 & $0,1,2$ & \\
\hline
\end{tabular}

Table 2. Interpretation of N-gain scores (Hake, 1998)

\begin{tabular}{lc}
\hline$\langle g\rangle$ score & Criteria of improvement of RCGP \\
\hline$\langle g\rangle \geq .7$ & High \\
$.3 \leq\langle g\rangle<.7$ & Medium \\
$\langle g\rangle<.3$ & Low \\
\hline
\end{tabular}

In both experimental and control groups, the instruction was conducted in the form of live lectures. The lecturers introduced implicitly some reading strategies (e.g., trying to prove before reading, identifying proof framework, breaking the proof in parts, using examples, comparing proofs) when reading proofs.

\section{Instrument for Measuring RCGP}

This study used as pre-test and post-test an identical RCGP test which was adapted from Yang and Lin's (2008) RCGP test items. The adaptation was based on the level of participants and the content of the course, and included the following: (1) some closed questions were changed into open questions, (2) the proposition presented in the test and its proof was adapted to the course content: congruent triangles, (3) proofs were presented in two-column and flow-chart formats, and (4) more formal terms were used, such as axiom and definition. The adapted version of RCGP is included in Appendix 1. The test took 30 minutes for PMT to complete. Quantitative measures regarding the five facets of RCGP were derived from all corresponding items, as shown in Table 2. The Cronbach's alpha reliability coefficient of the instrument for the total number of PMT was 0.703 .

The structure of the test including the operational definition of each facet is shown in Table 1. Except for items 1, 3, 4, 6, 7 and 10, all questions are answered by yes or no, or agree or disagree. Questions 3 and 5 ask for additional explanations, and partial scores of 1 and 2 points were given. For instance, questions 2 and 12 ask for PMT explanations for agreement or disagreement. If PMT answers were correct and their explanations were valid or relevant, students would get score 2 . If PMT answers were correct, but the explanations were not correct, then they got score 1 .

\section{Scoring and Analysis}

The first author scored the PMT answers, discussing boundary cases with the other two authors to agree on uncertain scores. A new scorer/assessor (properly trained) who had not participated in the prior scoring activities independently coded $25 \%$ of the data using a coding guide. We calculated Cohen's kappa to determine the inter-rater agreement between assessors $(\mathrm{\kappa}=.906)$.

We calculated normalized gains (N-gain) to determine the improvement of PMT performance for the whole RCGP and each facet of RCGP before intervention (pre-test) and after intervention (post-test) for both the experimental and the control group. We used Hake's formula (2002) to determine the N-gain score $\langle g\rangle$ for the difference between pre- and post-test scores. We used Mann-Whitney U test as a nonparametric test to examine the significance of differences between $\mathrm{N}$-gains of the experimental and control group.

The criteria for the improvement of PMT RCGP based on N-gain scores are shown in Table 2.

We used the procedure by Lin and Yang (2007) to determine PMT RCGP levels by assigning PMT RCGP scores with a vector triple $(a, b, c)$. The values of $a, b$, and $c$ refer to, respectively, the scores of facets of basic knowledge, logical status and summarization, and generality and application (Figure 1). Table 1 indicates that the total scores of the three values of $a, b$ and $c$ could be 4,5 and 7 , respectively. If a student scored above $70 \%$ in the basic knowledge items or 3 , the value of $a$ was 1 . In the same way, values of $b$ and $c$ for logical status and summarization, and generality and application, are determined. This means that the scores of $a, b$, and $c$ are either 0 or 1 . The PMT RCGP scores were classified into five categories: Beyond Chaining Elements $(1,1,1)$, Beyond Recognizing Elements $(1,1,0)$, Beyond Surface $(1,0,0)$, Surface $(0,0,0)$, and Skipping Recognizing- 
Table 3. Mean RCGP scores, N-gains and standard deviations (SD) of pre-test and post-test

\begin{tabular}{lccccccc}
\hline \multirow{2}{*}{ Groups } & \multicolumn{2}{c}{ Pre-test } & \multicolumn{2}{c}{ Post-test } & \multicolumn{2}{c}{ N-gain } & \multirow{2}{*}{ Criteria } \\
\cline { 2 - 7 } & Mean & SD & Mean & SD & Mean & SD & High \\
Experimental $(\mathrm{N}=60)$ & 7.25 & 2.26 & 13.75 & 1.781 & .733 & .219 & Hed \\
Control $(\mathrm{N}=65)$ & 7.80 & 2.51 & 10.80 & 2.425 & .330 & .309 & Medium \\
\hline
\end{tabular}

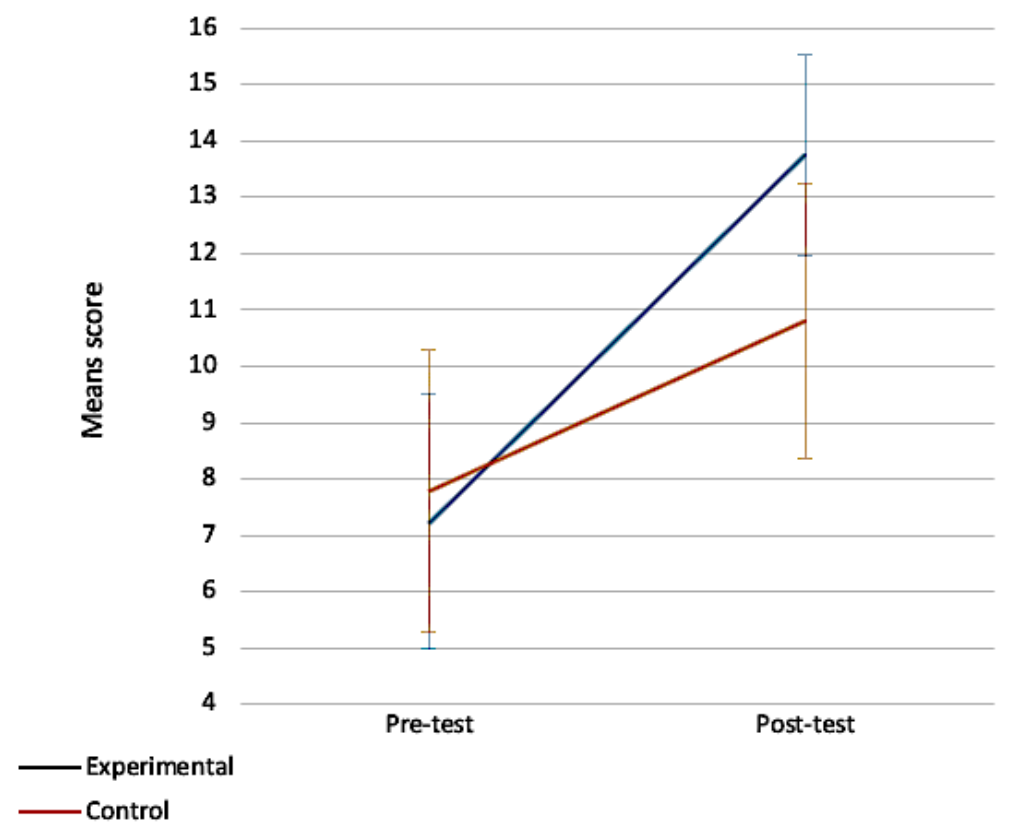

Figure 4. Plots of means of RCGP scores and standard deviations of pre-test and post-test in experimental and control groups.

Chaining $(1,0,1)$. The other possible responses: $(0,1,1)$, $(0,0,1)$, and $(0,1,0)$ were labelled as unsupported as we assume that one needs to comprehend the first facet (basic knowledge) before moving from the surface level to the next level.

\section{FINDINGS}

At the beginning of this section, we present descriptive statistics (i.e., mean and standard deviations) of RCGP scores of pre-test, post-test, as shown in Table 3. The pre- and post-test scores represent respectively PMT performance of RCGP before and after the intervention. The $\mathrm{N}$-gain scores represent the improvement of PMT RCGP performance after intervention. We compared PMT RCGP performance as an effect of the intervention setting in experimental and control groups by observing the significant difference of PMT performance between both experimental and control groups at the following two stages: before (pre) and after (post) intervention, and at the level of their improvement (N-gain).

Figure 4 presents plots of the means of RCGP scores of pre- and post-test in both experimental and control groups.

We found that the normalized gain of RCGP in the experimental group was $0.733(M=13.75)$, categorized as high, the normalized gain of the control group was $0.33(M=10.80)$, which was categorized as medium.
The Mann-Whitney $U$ test indicated that the pre-test mean scores for the experimental and control group did not differ significantly, $U(60,65)=1647$, two-tailed $p=$ 0.131 . The Mann-Whitney $U$ test indicated that the Ngain scores for experimental and control group differed significantly, $U(60,65)=547.5$, two-tailed $p<0.001$. Based on N-gains and the Mann-Whitney U tests, we drew the conclusion that the instruction with the use of flow-chart format together with other formats (e.g., paragraph and two-column proof formats) highly improves PMT RCGP.

Next, we also categorized the level of RCGP of PMT in both experimental and control groups. We used the comprehension categories proposed by Lin and Yang (2007). The aim of this analysis was to investigate the PMT level of RCGP based on the post-test scores representing PMT performance of RCGP after the intervention. We present the percentages of PMT who answered at least 70\% correctly of the items of the RCGP facets at the different levels, as shown in Table 4 . The comprehension categories in Table 4 indicate the level of RCGP reached by the PMT. So, the percentage of PMT who were in the "Beyond Chaining Elements" category indicated the percentage of PMT who reached the third or higher level of RCGP.

PMT performance on the post-test indicated that $56.7 \%$ of PMT in the experimental group reached beyond the third level of RCGP (Chaining Elements) while only 
Table 4. Percentages of PMT reaching 70\% in RCGP, based on the post-test scores

\begin{tabular}{|c|c|c|}
\hline Comprehension Category & Control $(n=65)$ & Experimental $(n=60)$ \\
\hline Beyond Chaining Elements $(1,1,1)$ & 6.2 & 56.7 \\
\hline Beyond Recognizing Elements and under Chaining Elements $(1,1,0)$ & 3.1 & 5 \\
\hline Skipping Recognizing-Chaining $(1,0,1)$ & 46.2 & 25 \\
\hline Beyond Surface and under Recognizing Elements $(1,0,0)$ & 18.5 & 5 \\
\hline Surface $(0,0,0)$ & 9.2 & 1.7 \\
\hline Unsupported responses $(0,1,0) /(0,0,1) /(0,1,1)$ & 16.9 & 6.7 \\
\hline Total & 100.0 & 100.0 \\
\hline
\end{tabular}

Table 5. Means and standard deviations of pre-test, post-test and N-gain of five facets of RCGP

\begin{tabular}{|c|c|c|c|c|c|c|c|c|}
\hline \multirow{2}{*}{ Facets } & \multirow{2}{*}{ Groups } & \multicolumn{2}{|c|}{ Pre-test } & \multicolumn{2}{|c|}{ Post-test } & \multicolumn{2}{|c|}{$\mathrm{N}$-gain } & \multirow{2}{*}{ Category } \\
\hline & & Means & SD & Means & SD & Means & SD & \\
\hline \multirow[t]{2}{*}{ Basic Knowledge } & Experimental & 2.283 & .958 & 3.533 & .596 & .744 & .343 & High \\
\hline & Control & 2.815 & .827 & 3.246 & .884 & .347 & .722 & Medium \\
\hline \multirow[t]{2}{*}{ Logical status } & Experimental & .433 & .593 & $2.400^{*}$ & .588 & $.660^{*}$ & .322 & Medium \\
\hline & Control & .569 & .661 & 1.292 & .805 & .270 & .401 & Low \\
\hline \multirow[t]{2}{*}{ Summarization } & Experimental & .567 & .673 & $1.400^{*}$ & .827 & $.660^{*}$ & .527 & Medium \\
\hline & Control & .277 & .573 & .400 & .725 & .041 & .440 & Low \\
\hline \multirow[t]{2}{*}{ Generality } & Experimental & 2.000 & 1.074 & 2.867 & .468 & .833 & .458 & High \\
\hline & Control & 2.046 & 1.152 & 2.708 & .631 & .923 & .230 & High \\
\hline \multirow[t]{2}{*}{ Application } & Experimental & 1.967 & .991 & 3.550 & .649 & .753 & .454 & High \\
\hline & Control & 2.092 & 1.100 & 3.154 & 1.093 & .579 & .502 & Medium \\
\hline
\end{tabular}

$6.2 \%$ of PMT in the control group reached this level. Beyond the third level of RCGP (Chaining Elements), PMT comprehended the five facets of RCGP; they were able to recognize proof elements (e.g., premises, properties, and a conclusion), understand their logical chaining in a proof, identify what is validated by the proof and apply the latter in other contexts. In the experimental group, $61.7 \%$ of prospective mathematics teachers reached the second or higher level of RCGP (Recognizing Elements), while only 9.3\% of the PMT from the control group was in that level. Beyond the "Recognizing Elements" level, PMT comprehended the first three facets of RCGP (e.g., basic knowledge, logical status and summarization) and they were able to recognize premises, conclusions, or properties that may be implicitly applied in a proof. These percentages show that the PMT in the experimental group reached higher RCGP levels than PMT in the control group.

The highest percentage of $46.2 \%$ of PMT in the control group was in the category of "Skipping RecognizingChaining" while $25 \%$ of PMT in the experimental group was in this category. These PMT could answer correctly at least $70 \%$ of the items related to the generality and application facets but the percentage of correct answers of items regarding logical status and summarization was less than $70 \%$. This indicated that a large part of the PMT was able to apply the proposition of the proof and identify what is validated by the proof but lacked the ability of recognizing the status of the statements (e.g., premises, conclusion and applied properties) in proof and the ability of identifying the critical ideas of proof (e.g., justification of the conclusion).

Lastly, we present descriptive statistics (i.e., means and standard deviations) of pre- and post-test, and N- gain scores of the five facets of RCGP (i.e., Basic Knowledge, Logical Status, Summarization, Generality and Application), as shown in Table 5. Through the analysis of these data, we wanted to investigate which facet of RCGP is supported by the use of multiple proof formats. We compared these scores for each facet and observed the significant differences in both experimental and control groups.

N-gains as shown in Table 5 indicated that the improvement of PMT RCGP performance of facets of basic knowledge, generality and application were high in the experimental group. The improvements of RCGP of the facets of logical status and summarization in the experimental group were medium, but higher than in the control group (see "Low" category in Table 5). The post-test mean score for basic knowledge for the experimental group did not differ significantly, $U(60,65)=1635.5$, two-tailed $p=.083$, from that for the control group. It indicated that, after the intervention, the understanding of terms, symbols and statements in proof for the experimental group was similar with that for the control group.

The Mann-Whitney $U$ test indicated that the N-gain scores of the facets of logical status and summarization for both groups differed significantly, $U(60,65)=536.5$, two-tailed $p<0.001$ and $U(60,65)=833.0$, two-tailed $p<0.001$, respectively. We concluded that there was a significant improvement on PMT performance in both logical status and summarization for the experimental group after the intervention. In other words, the instruction in the experimental group supported PMT to gain better RCGP performance in recognizing logical status of statements in the proof and critical ideas of the proof. We did not find differences in improvement of 
reading comprehension between experimental and control group on the other facets.

\section{DISCUSSION AND CONCLUSIONS}

This current study compared the effects of the use of multiple formats of presenting geometry proof on prospective mathematics teachers' reading comprehension of geometry proof (RCGP). We conducted an experimental design with a control group $(n=65)$, which only used the two-column format of geometry proof, and an experimental group $(n=60)$, which used three different formats (paragraph, twocolumn and flow-chart proof). At the beginning of the instruction, PMT performance of RCGP for the experimental and control groups was not significantly different. While the improvement, expressed as N-gain, of PMT RCGP in the experimental group was high, the control group's improvement was in the medium category. These findings suggest that the instruction using multiple proof formats had a stronger effect on PMT RCGP than instruction using one type of proof format (i.e., two-column proof), even though the geometry proof instruction using multiple proof formats was given to the PMT in just three 150-minute meetings.

We found that while $61.7 \%$ of PMT in the experimental group reached beyond the second level of RCGP "Recognizing Elements", only 9.3\% of PMT in the control group was beyond this level. In order to reach this level or beyond, PMT should be able to understand most of the terms and sentences in the proposition and its proof, to identify the status of statements such as premises, conclusions or applied properties, and also to grasp the critical ideas of the proof. The "logical status" and "summarization" facets contributed significantly to the difference found between the experimental and control groups, whereas PMT performance on the "basic knowledge" facet did not differ significantly between the groups. In particular, the improvement of these two facets (i.e., "logical status" and "summarization") in the experimental group justified the conclusion that using multiple proof formats better supported PMT in reaching a higher level of RCGP than a single proof format (two-column proof). This finding agreed with the suggestion made by Cirillo and Herbst (2011) that lecturers should introduce and give students opportunities of using different formats of proofs to facilitate their comprehension of a proof.

Several explanations may be given for the different gains in the facets of "logical status" and "summarization" between the two groups. First, the experimental group performed better in reading comprehension on the facet of "logical status", because the flow-chart proof format visualizes the logical status of propositions (e.g., premises, conclusion, applied properties/axioms/theorems/definitions) in the proof (Miyazaki et al., 2015). Second, the flow-chart format helped PMT recognize critical ideas of the proof. The critical ideas of proof refer to the connections between the intermediate or final conclusion and the previous statements or premises. The flow-chart visualizes this connection by the arrows between premises, intermediate conclusion, final conclusion and the reasons or applied properties (e.g., axioms, theorems, definitions), justifying the conclusion or claim (Cirillo \& Herbst, 2011; Miyazaki et al., 2015). In contrast, there was no significant difference in the post-test scores between the two groups related to the facets of "basic knowledge", "generality" and "application", although both groups progressed. The reason for this may be that PMT in both groups were instructed to solve writingoriented tasks, which provided PMT with application questions for practice. The use of multiple proof formats in the experimental group did not make a difference in "basic knowledge", "generality" and "application", because these only focused on presenting a certain proof, how the elements of the proof (i.e., premises, conclusion, and applied properties) were logically connected, and they did not connect with other propositions or other context.

Yang and Lin's model supposes that students' RCGP develops from "Surface" to "Recognizing Elements" then to "Chaining Elements", and finally to "Encapsulation" (Yang \& Lin, 2008). Also, this sequence is generally seen in geometry textbooks starting with proposition, then proof, and then its application (Lin \& Yang, 2007). However, the data analysis showed that $46.2 \%$ of the PMT in the control group and $25 \%$ of the PMT in the experimental group were in the "Skipping Recognizing-Chaining" category $(1,0,1)$. This indicates that they had good scores for "application" and "generality" but they scored low on "logical status" and "summarization". In other words, the PMT understood the mathematical terms or concepts in the proof and what is validated by the proof, and could apply the proposition properly in other, similar situations, but they did not understand the logical relations of the proofs and some critical ideas of the proofs. This was also found by Lin and Yang (2007). There may be several possible reasons for this finding. First, the PMT in both groups were instructed not only to read a proof (readingoriented tasks) but also to write a geomety proof (writing-oriented tasks). In writing proof tasks, PMT constructed a proof of a certain proposition by applying related theorems and propositions. To do so, it seems to us that PMT tried to understand which proposition should be proven by the theorems and how to apply the proposition to other contexts. These activities refer to the facets of "generality" and "application". Consequently, their reading may only have focused on what is validated by the proof and how to apply it in other contexts instead of understanding how each step followed from the previous one (Weber \& Mejia-Ramos, 2011). That may have enabled the PMT to comprehend 
the facets of "generality" and "application", but understanding of the facets of "logical status" and "summarization" was not necessary. So, we found that students' proof comprehension within the facets of "generality" and "application" could benefit from attempts in proof construction. Second, the lack of understanding of the PMT in the categories of "logical status" and "summarization" is related to a type of understanding which is called "instrumental" (Skemp, 1976). PMT with this type of understanding know how to apply propositions but show ignorance when it comes to how a proof validates a proposition. So, PMT might reach the "Recognizing" and "Chaining" level after they know how to read a proof (reaching "beyond chaining elements" level). However, such an explanation requires further studies on investigating the process of the development of RCGP over time (e.g., a learning trajectory of reading comprehension). Finally, our findings agreed with Lin and Yang (2007) that their RCGP model is not hierarchical as the succession of the students' attainment of RCGP levels is not always linear.

We are aware that our study has some possible limitations. The first one is the nature of the test we used. The items of RCGP used in this study were adapted from Yang and Lin's items (2008) by changing the type of some questions and reducing the number of questions. The Cronbach's alphas of pre-test and post-test (i.e., 0.624 and 0.705 respectively) indicate that the reliability of the pre-test and post-test are questionable and acceptable, respectively. However, the adaptation from Yang and Lin's items was minor, so we suppose that this did not significantly affect our results. A second limitation is that in our experimental design the experimental and control groups did not only differ in types and number of proof formats, but also in other conditions, like: (1) the use of Dynamic Geometry System (DGS) used in the experimental group to help PMT understand definitions, axioms or theorems, (2) the reading strategies that were introduced by the teacher, and (3) the role of the teacher (e.g., experience, use of teaching strategies). We supposed that the use of the DGS might affect PMT basic knowledge in the experimental group but our results show that the pretest scores of both groups in this facet were similar. For the other two factors (reading strategies and the role of the teacher) we do not have enough information to say if these factors may contribute to PMT RCGP.

A contribution of this paper to teaching practice is our finding that PMT reading comprehension can be improved by using a flow-chart proof format combined with other proof formats (two-column proof and paragraph proof) in proof reading tasks. Particularly, the flow-chart proof format could help PMT recognize the logical connection of the components in the proof (e.g., premises, conclusion, applied properties). This issue is relevant to the Indonesian context because most of the geometry proofs in geometry textbooks used in
Indonesia are presented in the two-column format. Also, in other contexts, we think that teaching students to read geometry proofs using multiple formats such as flowchart, paragraph and two-column proof may foster their RCGP. This paper also suggests that it may be beneficial to combine reading-oriented tasks and writing-oriented tasks to support students' reading comprehension. The reading-oriented tasks could help students comprehend the local aspects of proof (e.g., understanding statements, recognizing the logical status of statements, and the critical ideas of the proof) and the writingoriented tasks could support students in applying a proposition and its proof in other contexts.

The results of our current study suggest two recommendations for future research. First, in the context of students' progression of RCGP, the scores of the "Skipping Recognizing-Chaining" category in both groups indicated that many PMT understood the mathematical terms or concepts in the proof and what the proof validated and could apply the statement in similar situations but did not fully understand the logical relations among the arguments in the proof and some critical ideas of the proof. A possible interpretation is that the PMT development of understanding follows the relational understanding (Skemp, 1976), meaning that PMT could come to know the logical status and critical ideas of proof after they know how to apply the proof (Lin \& Yang, 2007). However, this study did not investigate the progress of PMT development over time. We suggest future studies investigating ways of students' development of RCGP. A model for describing the growth of students' reading comprehension by Ahmadpour et al. (2019) can be used to describe the transition between different stages of comprehension when reading a proof. This hypothetical model offers three different pathways of comprehending a formally acceptable proof, namely the path of structure, the path of the procedure, and the path of form. The end state of the first path, path of structure, is a formulated proof, meaning that the structure of proof has been understood. This path involves three transitions: generalization (i.e., process of applying a given argument in a broader context), abstraction (i.e., a process occurring when the subject focuses on specific properties of a given object and then considers this property in isolation from the original) and formalization (i.e., a process linking directly the text of a proof to the abstract structure). The second path, path of procedure, is a part of the first path: it branches off the first path by skipping the abstraction and by applying the formalization to a general procedure (i.e., reading proof as a general procedure). The last path, path of form, leads to seeing a formally acceptable proof as an argument that is correctly manipulated by symbols. In this case, students check the correctness of the proof by checking the correctness of each operation in an argument. The first two paths, structure and procedure, focus on the understanding of 
proof at the semantic level, and the last path, form, focuses on the use of symbolic representations at the syntactic level. Secondly, although we confirmed the role of formats of presenting geometry proofs in fostering students' RCGP, other factors should be further investigated, for instance students' reading strategies of a certain proof format. Pape (2004) states that students' reading strategies for comprehending proofs can be classified and explored with different propositions and within proof formats. Future research could investigate the relationship between RCGP performance and students' strategies to read proofs presented in multiple proof formats so that researchers and mathematics teacher educators gain insights into the students' perspectives and their strategies that may help with reading comprehension of geometry proof.

Author contributions: All authors have sufficiently contributed to the study, and agreed with the results and conclusions.

Funding: Funding for this study was provided by the Islamic Development Bank (IsDB) Project 4 in 1, Kementerian Pendidikan dan Kebudayaan (Kemendikbud), through Award IDN-1008.

Declaration of interest: No conflict of interest is declared by authors.

\section{REFERENCES}

Ahmadpour, F., Reid, D., \& Reza Fadaee, M. (2019). Students' ways of understanding a proof. Mathematical Thinking and Learning, 21(2), 85-104. https:/ / doi.org/10.1080/10986065.2019.1570833

Brandell, J. (1994). Helping students write paragraph proofs in geometry. Mathematics Teacher, 87(7), 498502. https://doi.org/10.5951/MT.87.7.0498

Cirillo, M., \& Herbst, P. G. (2011). Moving toward more authentic proof practices in geometry. Mathematics Educator, 21(2), 11-33.

Hake, R. R. (1998). Interactive-engagement versus traditional methods: A six-thousand-student survey of mechanics test data for introductory physics courses. American Journal of Physics, 66(1), 64-74. https://doi.org/10.1119/1.18809

Hake, R. R. (2002). Relationship of individual student normalized learning gains in mechanics with gender, high-school physics, and pretest scores on mathematics and spatial visualization. Physics Education Research Conference, 8(1), 1-14.

Herbst, P. G. (2002). Establishing a custom of proving in American school geometry: Evolution of the twocolumn proof in the early twentieth century. Educational Studies in Mathematics, 49(3), 283-312. https:/ / doi.org/10.1023/A:1020264906740

Inglis, M., \& Aberdein, A. (2015). Beauty is not simplicity: An analysis of mathematicians' proof appraisals. Philosophia Mathematica, 23(1), 87-109. https://doi.org/10.1093/philmat/nku014

Knuth, E. J. (2002). Teachers' conceptions of proof in the context of secondary school mathematics. Journal of
Mathematics Teacher Education, 5, 61-88. https://doi.org/10.1023/A:1013838713648

Lin, F. L., \& Yang, K. L. (2007). The reading comprehension of geometric proofs: The contribution of knowledge and reasoning. International Journal of Science and Mathematics Education, 5(4), 729-754. https://doi.org/10.1007/ s10763-007-9095-6

Mejia-Ramos, J. P., Fuller, E., Weber, K., Rhoads, K., \& Samkoff, A. (2012). An assessment model for proof comprehension in undergraduate mathematics. Educational Studies in Mathematics, 79(1), 3-18. https:// doi.org/10.1007/s10649-011-9349-7

Ministry of Education and Culture. (2013). Act Ministry of Education and Culture No. 68 of 2013 about Content Standard for Junior Secondary School. Retrieved from https://1ldikti12.ristekdikti.go.id/ 2013/06/28/ permendikbud-tentang-kerangkadasar-dan-struktur-kurikulum-tahun-2013.html

Ministry of Education and Culture. (2016). Act Ministry of Education and Culture no. 21 of 2016 about Content Standard for Secondary School. Retrieved from https://bsnp-indonesia.org/wp-content/ uploads/2009/06/Permendikbud_Tahun2016_No mor021_Lampiran.pdf

Miyazaki, M., Fujita, T., \& Jones, K. (2014). Functions of open flow-chart proving in introductory lessons of formal proving. In P. Liljedahl, S. Oesterle, C. Nicol \& D. Allan (Eds.), Proceedings of the Joint Meeting of the 38th Conference of the International Group for the Psychology of Mathematics Education and the 36th Conference of the North American Group for the Psychology of Mathematics Education (Vol. 4, pp. 225232). Vancouver, Canada: PME.

Miyazaki, M., Fujita, T., \& Jones, K. (2015). Flow-chart proofs with open problems as scaffolds for learning about geometrical proofs. ZDM - Mathematics Education, 47(7), 1211-1224. https://doi.org/ $10.1007 /$ s11858-015-0712-5

Miyazaki, M., Fujita, T., \& Jones, K. (2017). Students' understanding of the structure of deductive proof. Educational Studies in Mathematics, 94(2), 223-239. https:// doi.org/10.1007/s10649-016-9720-9

National Education Standard Board. (2020). Fokus Pembelajaran SD/MI, SMP/MTs, SMA/MA [Focuses of Learning in Elementary and Secondary School]. In W. Kamdi \& B. Suryadi (Eds.), Badan Standar Nasional Pendidikan. Retrieved from https:/ / bsnp-indonesia.org/wpcontent/uploads / 2020/12/Fokus-Pembelajaran-2.pdf

Panse, A., Alcock, L., \& Inglis, M. (2018). Reading proofs for validation and comprehension: An expertnovice eye-movement study. International Journal of Research in Undergraduate Mathematics Education, 
4(3), 357-375. https:/ / doi.org/10.1007/s40753-0180077-6

Pape, S. J. (2004). Middle school children's problemsolving behavior: A cognitive analysis from a reading. Journal for Research in Mathematics Education, 35(3), 187-219. https://doi.org/10.2307/ 30034912

Pereira-Laird, J. A., \& Deane, F. P. (1997). Development and validation of a self-report measure of reading strategy use. Reading Psychology, 18(3), 185-235. https:// doi.org/10.1080/0270271970180301

Rota, G. (1997). The phenomenology of mathematical beauty. Synthese, 111(2), 171-182. https:/ / doi.org/10.1023/A:1004930722234

Roy, S., Alcock, L., \& Inglis, M. (2010). Undergraduates proof comprehension: A comparative study of three forms of proof presentation [Paper presentation]. Proceedings of the 13th Conference on Research in Undergraduate Mathematics Education. Retrieved from http:/ / mathed.asu.edu/crume2009/ proceed ings.html

Selden, A. (2012). Transitions and proof and proving at tertiary level. In G. Hanna \& M. De Villiers (Eds.), Proof and Proving in Mathematics Education: The 19th ICMI Study (Vol. 15, pp. 391-420). Heidelberg: Springer. https://doi.org/10.1007/978-94-0072129-6_17

Selden, A., \& Selden, J. (2017). A comparison of proof comprehension, proof construction, proof validation and proof evaluation. In R. Göller, R. Biehler, R. Hochmuth, \& H. G. Rück (Eds.) Proceedings of kompetenzzentrum hochschuldidaktik mathematik Conference (pp. 339-345). Hanover, Germany: khdm.
Skemp, R. R. (1976). Relational understanding and instrumental understanding. Mathematics Teaching, 77, 20-26. https://doi.org/10.5951/MTMS.12.2. 0088

Weber, K. (2009). Mathematics majors' evaluation of mathematical arguments and their conception of proof. In Proceedings of the 12th Conference for Research in Undergraduate Mathematics Education. Retrieved from http://sigmaa.maa.org/rume/ crume2009/ proceedings.html

Weber, K., \& Mejia-Ramos, J. P. (2011). Why and how mathematicians read proofs: An exploratory study. Educational Studies in Mathematics, 76(3), 329-344. https:// doi.org/10.1007/s10649-010-9292-z

Wong, W. K., Yin, S. K., Yang, H. H., \& Cheng, Y.-H. (2011). Using computer-assisted multiple representations in learning geometry proofs. Educational Technology and Society, 14(3), 43-54.

Yang, K. L. (2012). Structures of cognitive and metacognitive reading strategy use for reading comprehension of geometry proof. Educational Studies in Mathematics, 80(3), 307-326. https:// doi.org/10.1007/s10649-011-9350-1

Yang, K. L., \& Lin, F. L. (2008). A model of reading comprehension of geometry proof. Educational Studies in Mathematics, 67(1), 59-76. https:// doi.org/10.1007/s10649-007-9080-6

Yang, K. L., \& Lin, F. L. (2012). Effects of readingoriented tasks on students' reading comprehension of geometry proof. Mathematics Education Research Journal, 24(2), 215-238. https://doi.org/10.1007/ s13394-012-0039-2 


\section{APPENDIX 1}

As shown in Fig. $4, \overline{W Z}$ bisects $\angle T W V$ and $\overline{W T} \cong \overline{W V}$; then must $\overline{T Z}$ and $\overline{V Z}$ be congruent?

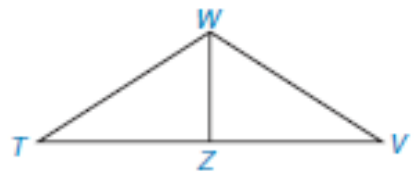

Figure 4.

For this problem, Budi makes a proof (Fig. 5):

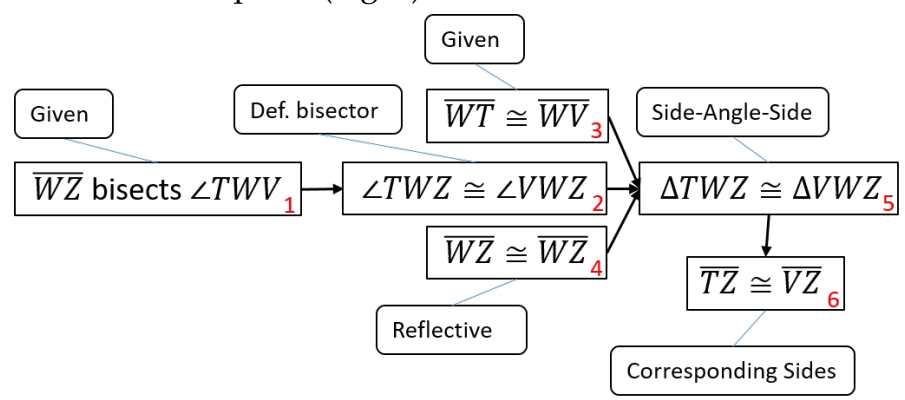

Figure 5. Flow-chart proof

\begin{tabular}{lll}
\hline Statements & Reasons & \\
$\overline{\overline{W Z} \text { is a bisector of an angle } \angle T W V}$ & Given & Line 1 \\
$\angle T W Z \cong \angle V W Z$ & Definition of a bisector of an angle & Line 2 \\
$\overline{W T} \cong \overline{W V}$ & Given & Line 3 \\
$\overline{W Z} \cong \overline{W Z}$ & Axiom of reflection & Line 4 \\
$\Delta T W Z \cong \Delta V W Z$ & Axiom Side-Angle-Side & Line 5 \\
$\overline{T Z} \cong \overline{V Z}$ & Definition of congruent triangles & Line 6 \\
\hline
\end{tabular}

Answer the following on the basis of the question "must $\overline{T Z}$ and $\overline{V Z}$ be congruent?" and Figure 5.

1. Label $\angle T W Z$ in Figure 4 as 1 and $\angle V W Z$ in Figure 4 as 2.

2. Do you agree that $\angle T W Z \cong \angle V W Z$ ? Explain why or why not.

3. If $\triangle T W Z \cong \triangle V W Z$, what is the corresponding side of $\overline{T Z}$ ?

4. Apart from the known conditions ( $\overline{W Z}$ bisects $\angle T W V$ and $\overline{W T} \cong \overline{W V}$ ), which conditions can be directly applied?

5. If someone suggests that the proof process of flow-chart 1,2,3, 4, 6, 5 is correct after box 5 and 6 are interchanged, would you agree with his or her opinion?

6. Which properties/axioms are applied to this proof?

7. This proof process derives an important result from the condition that $\overline{W Z}$ bisects $\angle T W V, \overline{W T} \cong \overline{W V}$ and another condition.

a) What is this important result?

b) What can be derived from this important result?

8. Do you agree that this proof process is correct?

9. Statement A: If $\overline{W Z}$ bisects $\angle T W V$ and $\overline{W T} \cong \overline{W V}$; then $\overline{T Z} \cong \overline{V Z}$.

a) Do you agree that this proof process can prove that Statement $\mathrm{A}$ is always correct?

b) Do you agree that this proof process can prove that Statement A is sometimes correct and sometimes incorrect? Answer the following questions on the basis of what you know.

10. In triangle $\triangle A B C, \overline{A B} \cong \overline{A C}$ and $\overline{A D}$ is an angle bisector of $\triangle A B C$, which conclusions can be derived?

11. There is a circle with center point $P$, radius $P Q$ and $P R$. If a point $S$ is an intersection point of a bisector of $\angle Q P R$ and $\overline{R Q}$, are $\overline{Q S}$ and $\overline{R S}$ congruent?

12. Sides $\overline{V W}$ and $\overline{V U}$ are legs of an isosceles triangle $\Delta V W U$ and a point $T$ is on the base $\overline{W U}$ such $\angle T V W \cong \angle T V U$, then is $\overline{T W} \cong \overline{T U}$ always correct?

The test is adapted from Yang and Lin (2008).

\section{http://www.ejmste.com}

